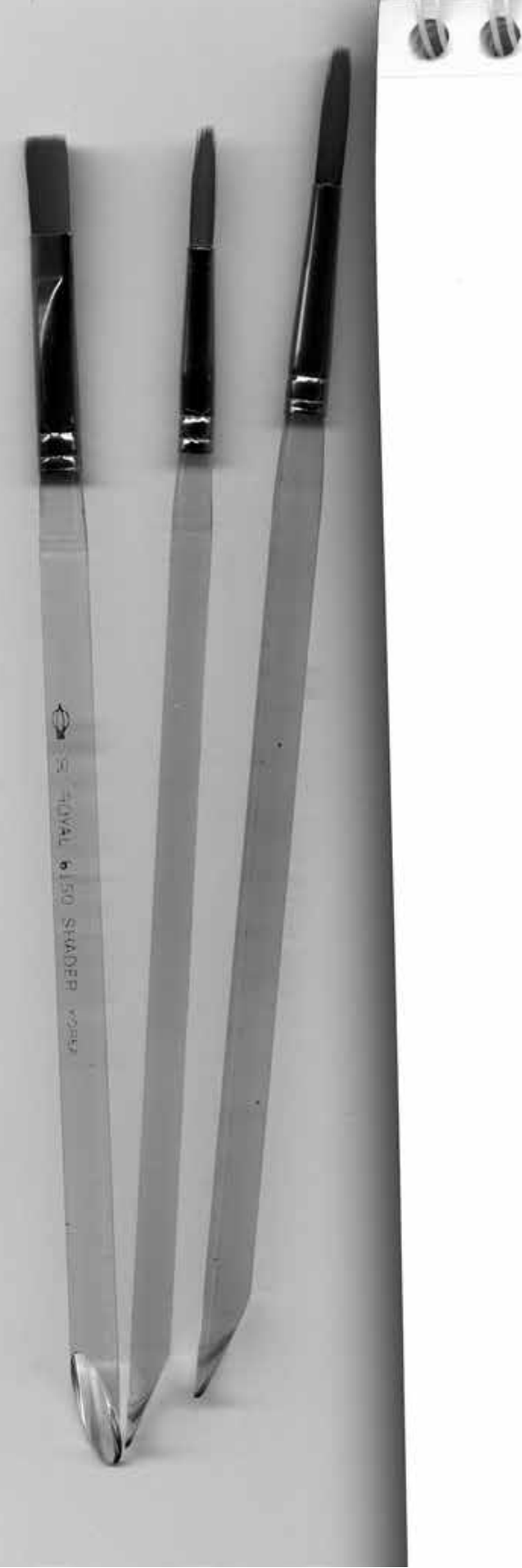

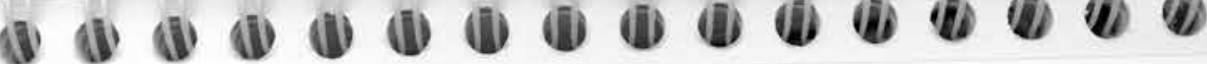
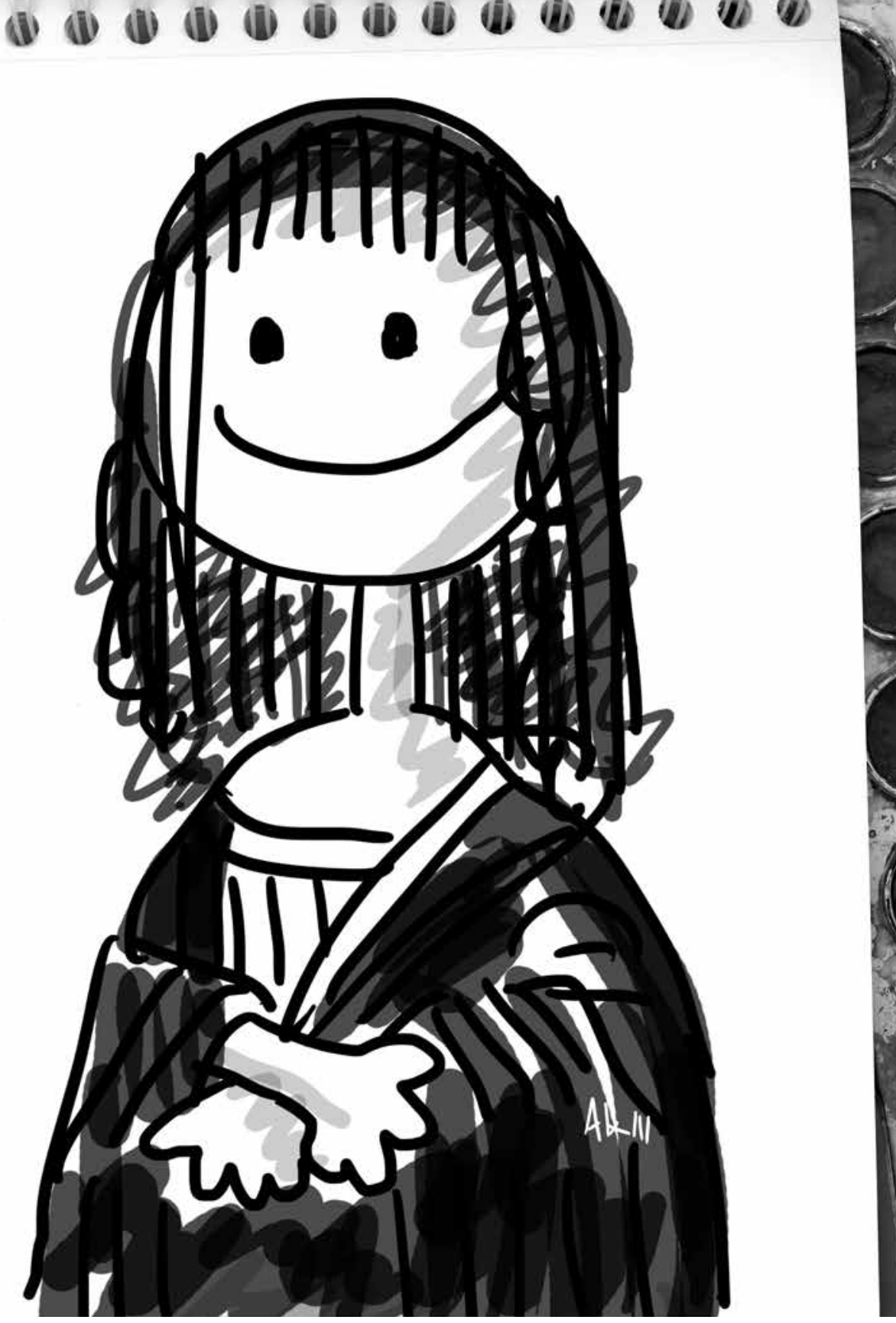

El arte en los Jardines infantiles de Bogotá: una experiencia de formación y acompañamiento a maestras de Educación Inicial

Santiago Barbosa Reyes José Ignacio Galeano Borda 
Santiago Barbosa Reyes ${ }^{1}$ José Ignacio Galeano Borda²
1 Maestro en Artes Plásticas, Universidad Nacional de Colombia. Candidato a Especialista en Creación Narrativa, Universidad Central. Profesional del Equipo Pedagógico de la Secretaría Distrital de Integración Social desde 2008. Parte del equipo constructor del Lineamiento Pedagógico para la Educación Inicial en el Distrito Capital. E-mail: barbosa santiago@yahoo.com

2 Fonoaudiólogo y Magíster en Educación, Universidad Nacional de Colombia. Docente catedrático Licenciatura en Educación Infantil, Universidad Pedagógica Nacional. Profesional del Equipo Pedagógico de la Secretaría Distrital de Integración Social desde 2007. Parte del equipo constructor del Lineamiento Pedagógico para la Educación Inicial en el Distrito Capital. E-mail: fonojoseignacio@yahoo.com

Fecha de recepción: 19 de febrero de 2013 / Fecha de aprobación: 24 de febrero de 2013

\section{El arte en los jardines infantiles de Bogotá: una experiencia de formación y acompañamiento a maestras de Educación Inicial}

\section{Resumen}

En este artículo se pretende realizar una presentación breve y concisa de la experiencia ocurrida entre los años 2008 y 2012, concerniente al trabajo de formación a maestras de jardines infantiles de la Secretaría Distrital de Integración Social (SDIS) de la ciudad de Bogotá, específicamente en lo referido al arte en la educación inicial; trabajo que ha sido liderado por el Equipo Pedagógico de la Subdirección para la Infancia de la SDIS. Se espera con este texto aportar en la comprensión del arte en la primera infancia, pero sobre todo difundir la existencia de experiencias de formación en arte desde una perspectiva pedagógica en la educación inicial.

\section{Palabras clave: Educación} Inicial, Arte, Experiencia pedagógica, Formación docente.

\section{Art in Bogotá kindergartens: experience of training and support for early education teachers}

\section{Summary}

This article aims to make a brief and concise presentation of the experience that occurred between 2008 and 2012 referred to the work of training teachers of kindergartens of the Secretaría Distrital de Integración Social (SDIS) of Bogotá, specifically referred to art in early childhood education; work has been led by the teaching staff of the Directorate for Children of the SDIS. This text is expected to contribute in the understanding of art in early childhood, but mostly spread the existence of training experiences in art from a pedagogical perspective in early childhood education.

Keywords: Early Childhood Education, Art, Teaching experience, teacher training.

\section{Arte em jardins de infância de Bogotá: uma experiência de formação e apoio a professores da educação infantil}

\section{Resumo}

0 presente artigo tem como objetivo fazer uma apresentação breve e conciso da experiência que ocorreu entre 2008 e 2012 , referiu-se ao trabalho de formação de professores de creches do Secretaría Distrital de Integración Social (SDIS) de Bogotá, especificamente com relação à arte na educação infantil, o trabalho foi conduzido pelo corpo docente da Direcção para as crianças da SDIS. Este texto é esperado para contribuir na compreensão da arte na infância, mas principalmente difundir a existência de experiências de formação em arte a partir de uma perspectiva pedagógica na educação infantil.

Palavras-chave: Educação Infantil, Arte, experiência de ensino, formação de professores. 


\section{Introducción}

$\mathrm{E}$ Equipo Pedagógico es un grupo de trabajo adscrito a la Subdirección para la Infancia de la Secretaría Distrital de Integración Social -SDIS- ${ }^{3}$, antiguo Departamento Administrativo de Bienestar Social-DABS- y hoy en día SDIS, bajo resolución desde 2007, que ha tenido dentro de sus principales líneas de acción el proceso de formación a maestras de los jardines infantiles que están a cargo de los niños y las niñas de primera infancia. Dicha formación ha contado con diferentes énfasis tanto temáticos como metodológicos, lo cual ha dependido del grado de desarrollo del concepto de educación inicial y del momento histórico en el cual se ha vivido este proceso.

La formación en arte ha tenido diferentes matices que han pasado, de un trabajo con poca presencia de sus componentes dentro de la práctica pedagógica de las maestras, a una ubicación central dentro de la misma. El sentido de este artículo pretende dar cuenta de dicho proceso, primero, para rescatar el papel del arte en la educación inicial desde una mirada pedagógica, y segundo, para comprender cómo éste ha sido abordado desde la institucionalidad.

Un momento importante en la formación a maestras en arte en la educación inicial, se ha dado desde las posibilidades que abre la construcción, y posterior posicionamiento, del Lineamiento Pedagógico para la Educación Inicial en el Distrito Capital $^{4}$, en tanto su estructura propone al arte, junto a la literatura, el juego y la exploración del medio, como uno de los pilares de este nivel educativo, y en ese sentido lo instaura como una de las actividades propias de la educación inicial.

3 La Secretaría Distrital de Integración Social, es una entidad pública de la ciudad de Bogotá encargada de liderar y articular la territorialización de política social para la disminución de la segregación, la garantía de los derechos y el reconocimiento y generación de oportunidades, y el ejercicio pleno de las ciudadanías. Para ampliar información al respecto, se recomienda consultar: www.integracionsocial.gov.co

4 El Lineamiento Pedagógico y Curricular para la Educación Inicial en el Distrito Capital es una elaboración realizada de manera conjunta entre la Secretaría Distrital de Integración Social y la Secretaría de Educación del Distrito de la ciudad de Bogotá y publicada en el año 2010. Presenta la apuesta pedagógica distrital para la educación dirigida a niños y niñas de cero a cinco años, especificando las particularidades educativas del nivel de educación inicial.
Aunque en el Lineamiento Pedagógico se asumen como pilares diferenciados el arte y la literatura, en tanto ambas experiencias son llamadas a conformarse como actividades propias para el trabajo con la primera infancia, esto no quiere decir que la literatura no forme parte de las experiencias de índole artística, pues constituye una ventana cultural para los niños y las niñas y comparte además las características propias de toda experiencia artística.

Es más, las más recientes apuestas políticas respecto a la educación inicial en Colombia ${ }^{5}$, consideran que la importancia del acercamiento al lenguaje en la primera infancia, la actividad propia de los niños y las niñas de jugar con el lenguaje, y el papel de la literatura infantil en el desarrollo infantil, justifica considerar a la literatura como un pilar de la educación inicial. No obstante, por las características tan específicas de este artículo y porque también consideramos además a la literatura como una experiencia artística, nos dedicaremos también a tratarla.

El artículo presenta en una primera parte tres hitos del proceso de formación: De la constitución del Equipo Pedagógico (1998) a la movilización de la propuesta del Proyecto Pedagógico del DABS (SDIS desde 2007); la construcción del Lineamiento Pedagógico y Curricular para la Educación Inicial en el Distrito (2008-2010); y el posicionamiento del Lineamiento Pedagógico (2010-2012). En la parte final, se recogen algunas proyecciones del trabajo de formación en arte y se presentan las conclusiones de este artículo.

\section{Momentos que recogen la experiencia}

\section{a. El Equipo Pedagógico (1998 - 2008)}

Si bien la conformación del Equipo Pedagógico tiene sus orígenes cuando la entidad aún se nominaba DABS en el año 1998, y a pesar de que no se puede desconocer la existencia

\footnotetext{
5 Nos referimos aquí al Lineamiento Pedagógico y Curricular para la Educación Inicial en Bogotá (2010, SDIS y SED) y al Documento Base para la Construcción del Lineamiento Pedagógico de Educación Nacional (2012, MEN).
} 
de algunos procesos de formación en arte, sí podemos afirmar que solamente hasta el año 2000 es posible ubicar documentalmente referencias directas al arte como uno de los aspectos a tener en cuenta en el trabajo con niños y niñas.

Parte de ello puede corroborarse en el documento Proyecto Pedagógico Red de Jardines Sociales publicado por el antiguo DABS (desde el 2007 SDIS) en el año 2000, y elaborado por el Equipo Pedagógico (SDIS, 2012), en donde la Expresión Artística se presenta como una línea de acción. En ésta se plantea el arte como un medio para elaborar las experiencias vividas durante la historia personal. De igual modo, se van a considerar como expresiones artísticas la música, pintura, literatura, danza y teatro, además de constituirse en vivencias únicas e irrepetibles para el desarrollo de la sensibilidad y la capacidad creadora (DABS, 2000).

Posterior a ello, y con la elaboración en el 2003 del documento Desarrollo Infantil y Educación Inicial, o libro naranja como se conoce en el ámbito de los jardines infantiles, el concepto de educación inicial va a ser mucho más visible y ganará en claridad. Este documento va a servir de base en la ulterior configuración y consolidación del Lineamiento Pedagógico, en donde se reestructura y reconceptualiza el lugar del arte en la educación inicial.

En el libro naranja se va a considerar al arte como un escenario de aprendizaje (DABS, 2003). Tal acepción se liga a las ideas del arte como una estrategia pedagógica que ayuda a propiciar diversos procesos en los niños y las niñas, y la del arte como un medio de expresión. La plástica, la música, la pintura, la poesía, la danza, el teatro y la literatura, van a ser considerados como lenguajes cercanos a los niños y niñas por medio de los cuales ellos y ellas se expresan.

La literatura, por su parte, va a ser considerada también como una estrategia para el desarrollo y como un campo de expresión capaz de potenciar el desarrollo en diferentes dimensiones (DABS, 2003). Se considera a la literatura como arte, pero se separa en tanto ocupa un lugar esencial en el Proyecto del DABS.

A diferencia de este ritmo en la construcción conceptual en torno al arte y la apuesta pedagógico-educativa de la entidad,

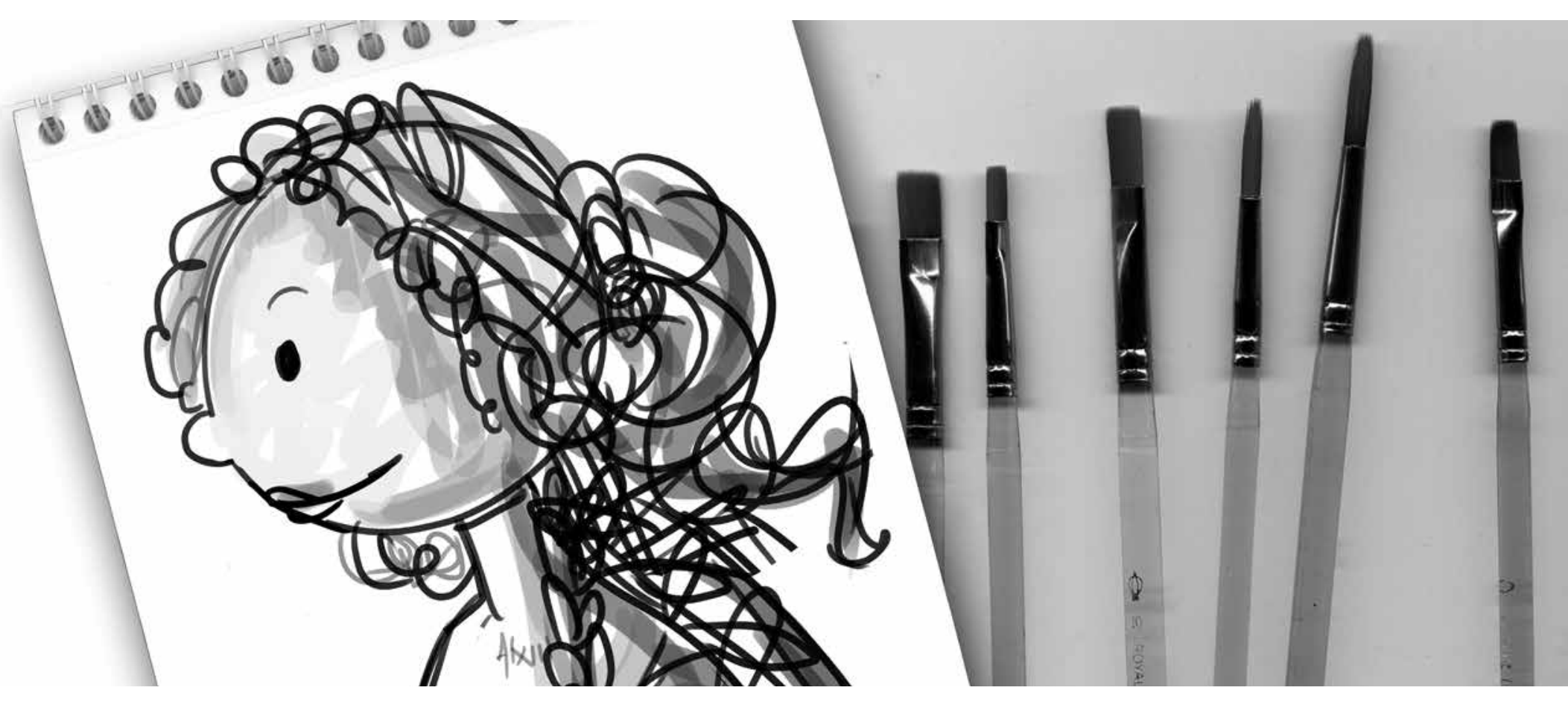

50 Educación y CiUdad NN24 Enero - Junio de 2013 ISSN 0123-0425 Pp. 47-58 


\section{El arte no aparece catalogado dentro de estas dimensiones, no es posible dar cuenta de procesos formativos vinculados con la idea del arte como algo propio del desarrollo o como una experiencia que lo potencia"}

los procesos de formación tuvieron otro ritmo en su desarro1lo. La práctica del viernes pedagógico ${ }^{6}$, que fue instaurada varios años atrás, permaneció, aunque no es posible desde la indagación realizada ubicar elementos concernientes al arte en estas jornadas. Contrario a ello, se pueden destacar diferentes procesos de formación como el desarrollado con la Fundación Batuta, en el que se le dio relevancia a la educación musical para niños y niñas (SDIS, 2012).

Puede explicar lo anterior, y desde la experiencia vivida por los autores del presente documento, el hecho de que el proceso de formación a maestras, entre los años 2004 y 2008, se centró de manera enfática en los procesos de desarrollo infantil ligados a la estructura de las dimensiones: corporal, comunicativa, lógica del pensamiento y personal social. Muestra de ello, fue el esfuerzo puesto en adelantar la construcción e implementación de la Guía de Observación, concebida como una herramienta que les permitiría a las maestras acceder a informaciones alrededor del desarrollo de los niños y las niñas. Esto se derivaría del enfoque de derechos implantado en la década del 2000.

En tanto el arte no aparece catalogado dentro de estas dimensiones, no es posible dar cuenta de procesos formativos vinculados con la idea del arte como algo propio del desarrollo o como una experiencia que lo potencia. Lo anterior puede ser

6 Para la SDIS (2012), el viernes pedagógico es un espacio con una tradición de más de veinte años, en el que jardines infantiles de la SDIS, en todas sus modalidades, no prestan atención directa niños y niñas, para que el equipo de trabajo del jardín infantil pueda realizar procesos de trabajo y cualificación docente. Lo anterior surge del interés de dotar de sentido pedagógico la permanencia de los niños y las niñas en los jardines infantiles, haciendo evidente la necesidad de ir más allá de brindarles cuidado y protección. Actualmente es un espacio que se desarrolla en jornada completa una vez al mes y se constituye en un escenario de encuentro que permite una articulación entre los planteamientos técnicos definidos por la Subdirección para la Infancia de la SDIS, y el trabajo específico de los equipos pedagógicos de los jardines infantiles (SDIS 2012). explicado por el evidente distanciamiento de una perspectiva pedagógica de la infancia para asumirla desde una mirada mucho más piscologizada, por lo que la preocupación inicial va a ser por los procesos de desarrollo y no por su potenciamiento. La formación de las maestras se centrará en el desarrollo más que en una pedagogía para la infancia.

\section{b. Construcción del Lineamiento Pedagógico (2008 - 2010)}

Hacia mediados del año 2008, el Equipo Pedagógico Central de la SDIS va a tener como principales objetos de trabajo: la formulación del Lineamiento Pedagógico para la Educación Inicial y, a la par de ello, el proceso de viernes pedagógico. Para cumplir con este ejercicio, se lleva a cabo un convenio interinstitucional entre la SDIS y la Universidad Pedagógica Nacional -UPN-7 , en el que se que se conforma un equipo integrado por algunos contratistas que venían del proceso anterior y otras personas con una rica experiencia y formación en pedagogía infantil. Adicional a ello, la presencia intencional de artistas en el equipo, un músico y un artista plástico, va a tener un impacto interesante en el desarrollo de estos objetivos.

La perspectiva de trabajo que adoptaría este nuevo equipo va a reconocer que las maestras de los jardines infantiles han ganado importantes saberes acerca del desarrollo infantil, necesarios en su ejercicio profesional, pero se va a enfatizar en el hacer y en los conocimientos prácticos de las maestras, respondiendo al qué hacer con un grupo de niños. Este giro hacia lo pedagógico va ser el derrotero de la formación a maestras; fue desarrollado en los viernes pedagógicos, y conceptos como las formas de trabajo pedagógico o las estrategias pedagógicas van a tomar una importante fuerza.

\footnotetext{
7 La Universidad Pedagógica Nacional es la universidad pública encargada de la formación de profesionales de la educación. Además, cumple funciones de investigación, producción y difusión de conocimiento profesional docente, educativo, didáctico y pedagógico, y contribuye a la formulación de políticas públicas
} 
Desde allí, el Trabajo por Proyectos, los Rincones de Trabajo, los Talleres y las Asambleas, constituirán el centro de la formación, estrategias que serán fundamentales para visibilizar el papel del arte en el trabajo con la primera infancia. De manera especial los Talleres para la educación inicial, que si bien no son una estrategia exclusiva para las artes, van a constituir un espacio pedagógico en el que se trabajan las artes con mayor insistencia, dada su premisa metodológica de aprender desde el hacer.

Esta organización en los procesos de formación permitiría un ambiente idóneo para la construcción del Lineamiento Pedagógico y su posterior posicionamiento e implementación. Como se anotaba, su construcción requirió de la presencia de diferentes profesionales, dentro de los que se incluyen algunos con formación y experiencia en artes (plásticas, música, literatura infantil), aspecto que va a ser clave, al reforzar una de las orientaciones para la realización del documento: El reconocimiento del arte como parte fundamental del quehacer pedagógico en Educación Inicial.

En ese trabajo, el arte y la literatura van a constituirse en pilares de la educación inicial (SDIS y SED, 2010), pues, en tanto hacen parte de las actividades propias de la infancia, posibilitan las relaciones consigo mismo, entre los niños y de éstos con los adultos y permiten tejer un puente con la cultura. Además, el arte pasa también a ser considerado una dimensión del desarrollo humano e infantil, apuntando a una concepción de desarrollo mucho más integral.

Como es posible observar, en el Lineamiento Pedagógico se amplía la mirada del arte en la educación inicial, en tanto que se organiza una estructura alrededor de qué trabajar pedagógicamente en arte y qué prácticas son las más adecuadas y pertinentes para potenciar el desarrollo infantil desde la experiencia artística. Estos planteamientos permitirán enriquecer de manera más amplia las prácticas con que se venía trabajando en los jardines, al considerar al arte no como un medio para aprender contenidos temáticos puntuales y de aprestamiento, sino como parte del desarrollo integral, derivado de vivir una experiencia valiosa y con sentido en la vida de los niños y las niñas.

Es importante tener en cuenta que antes del Lineamiento Pedagógico la mayoría de las prácticas que se venían dando con frecuencia desde el arte, en el trabajo con los niños y las niñas, se enmarcaban principalmente en dos vertientes; la primera, que se relaciona más con una aproximación a escenarios como las visitas a museos, la exploración de obras de arte y la asistencia a espectáculos, y la segunda, enfocada en la realización de actividades que debían su carácter "artístico" a la utilización de aquellos materiales considerados tradicionalmente como "artísticos", y en donde no siempre se reflexionaba realmente sobre el proceso artístico y su estrecha relación con el desarrollo infantil, ya que contaban con un fundamento apoyado casi que exclusivamente en lo manual (manualidades) y/o donde primaba el objetivo de conformar un producto predeterminado.

Si bien, de lo anteriormente nombrado, la aproximación a escenarios, espectáculos y obras de arte, son algunos de los aspectos importantes a tener en cuenta dentro de la manera como se pretendía involucrar al arte en las experiencias de los niños y las niñas, propuestos antes de la aparición del Lineamiento Pedagógico; sin embargo, es desde su aparición cuando se da inicio a una reflexión profunda sobre el sentido e intencionalidad de una experiencia artística en la que se quiso llegar a entender el valor de la práctica pedagógica vinculada al arte mucho más allá: como una experiencia propia de la primera infancia, inherente a lo humano y fundamental dentro del desarrollo infantil. Es decir, la idea de Pilar y de Dimensión del desarrollo bajo un abordaje pedagógico, lo cual permitiría dejar la noción del arte como método, instrumento o estrategia.

El concepto de educación inicial que se presenta en el Lineamiento Pedagógico, replantea el abordaje del qué y el cómo se lleva a la práctica el trabajo pedagógico con niños de 0 a 5 años, y dentro de dicha práctica pedagógica está comprendido el arte. El arte como pilar de la educación inicial y como dimensión del desarrollo infantil, permite su concepción como una experiencia necesaria en el trabajo con la primera infancia porque es uno de los aspectos del desarrollo infantil que conlleva circunscrito su potenciamiento.

Pero además, el arte está constituido por diferentes experiencias como la plástica, la música y la dramática, que pueden ser abordadas pedagógicamente a través de unos ejes de trabajo pedagógico que las caracterizan y son transversales a cada una de ellas: el sentido estético, la sensibilidad, la expresión y la 


\section{Dentro de los énfasis que mantenían o adoptarían varios jardines infantiles, muchos de ellos incluían el arte o la literatura infantil"}

creatividad (SDIS y SED, 2010). En ese orden de ideas, los ejes de trabajo pedagógico en el arte pueden ser entendidos como aquellos que conforman y caracterizan cualquier tipo de experiencia artística y que permiten a su vez estructurar el trabajo pedagógico, partiendo desde un sentido e intencionalidad pedagógicos más que desde un tema.

Por poner un ejemplo, es muy diferente abordar los colores como un tema teniendo únicamente como objetivo que los niños y las niñas los nombren o identifiquen, a que, como propone el Lineamiento Pedagógico, se parta de una claridad de sentido e intencionalidad pedagógica desde el potenciamiento de la creatividad, la sensibilidad, la expresión y el sentido estético, relacionando desde allí múltiples y posibles experiencias con el color.

Para cerrar este apartado se hace referencia, como anteriormente se enunció, al proceso que, paralelo a la construcción del Lineamiento Pedagógico, se vino realizando por el Equipo Pedagógico: el viernes pedagógico. Allí, además de lo descrito, un trabajo tuvo especial vinculación con la formación en arte, específicamente en la fase de finalización de la elaboración de este documento, se trata del acompañamiento a la reformulación de los proyectos pedagógicos de los jardines infantiles. Este acompañamiento tuvo como uno de sus antecedentes la construcción de un documento de orientaciones para la elaboración o revisión de los proyectos pedagógicos ${ }^{8}$, que pretendía orientar a los jardines infantiles al momento de dar revisión a su propuesta pedagógica y que fue enviado a cada institución.

A lo largo del segundo semestre de 2009 se inicia este proceso en los viernes pedagógicos, con el propósito de fortalecer cada proyecto pedagógico. Se partió de revisar lo que los jardines han construido para, a partir de ello, reflexionar sobre los componentes más relevantes en un proyecto pedagógico, apuntando a reconfigurar su sentido y estructura. Es relevante

8 Se habla del documento Orientaciones para la estructuración y/o revisión del proyecto pedagógico (SDIS, 2009). mencionar que dentro de los énfasis que mantenían o adoptarían varios jardines infantiles, muchos de ellos incluían el arte o la literatura infantil. De este modo, el acompañamiento a los jardines infantiles insistió en las características del trabajo pedagógico en arte, teniendo como referente el Lineamiento Pedagógico ya construido con las premisas anteriormente expuestas. Todo esto hizo parte del trabajo que prepararía el terreno en los jardines infantiles para la llegada del Lineamiento Pedagógico.

\section{c. Posicionamiento del Lineamiento (2010-2012)}

Posterior al lanzamiento del Lineamiento Pedagógico en el 2010, el trabajo del Equipo Pedagógico y del Equipo de Asesoría Técnica a Servicios Sociales en Primera Infancia - Componente pedagógico-, tendrá como una de sus líneas de trabajo el posicionamiento y la movilización social del documento. El primero desarrollaría este trabajo hacía fuera de la SDIS, mientras que el segundo trabajaría principalmente con jardines infantiles de la SDIS.

Se describirá a continuación lo que cada uno de estos equipos realizó en este periodo, referido a la formación en arte, la experiencia desde un nuevo convenio interinstitucional entre la SDIS y la UPN, y al final se comentará la consolidación del equipo que se encargaría de este proceso de manera específica.

\section{La experiencia del Equipo Pedagógico}

Como parte de su plan de trabajo, el Equipo Pedagógico desarrolló la estrategia de posicionamiento del Lineamiento Pedagógico en universidades que tuvieran licenciaturas de educación infantil, asociaciones que tuvieran que ver con la educación inicial y empresas que comerciaran y produjeran material didáctico para este nivel educativo.

De esta manera, se buscó socializar y posicionar el Lineamiento Pedagógico Curricular en el ámbito distrital y nacio- 
nal, generando escenarios de discusión que posibilitaran avanzar en los procesos pedagógicos de primera infancia y educación inicial, dentro de los que se incluía la apuesta pedagógica en torno al arte, así como promover el debate sobre el papel del ciclo de educación inicial dentro del sistema educativo en el marco de las políticas públicas por la primera infancia y educación inicial. Se contempló como ruta general la socialización del Lineamiento Pedagógico dando detalle de sus componentes estructurales en una presentación diseñada para tal fin, y mediante la realización de un diálogo de saberes con los colectivos anteriormente mencionados en torno al documento.

\section{Asesoría a Servicios Sociales de Infancia en el Estándar Proceso Pedagógico}

A partir de mediados de 2010, el Equipo de Asesoría a Servicios Sociales de Infancia en el Estándar Proceso Pedagógico, va a asumir el trabajo de formación pedagógica directamente con los jardines infantiles de la SDIS, teniendo como marco de referencia el Lineamiento Pedagógico. Este equipo va a desarrollar su trabajo para posicionar la apuesta pedagógica de la SDIS a través de tres estrategias: Acompañamiento, Formación y Actualización Permanente (dentro de la que se incluye el trabajo de Conferencias masivas), Viernes pedagógicos (SDIS, 2012). Por mayor pertinencia y trabajo alrededor del arte y la literatura se hará mención en este artículo a los viernes pedagógicos y las conferencias masivas.

\section{Viernes pedagógico}

En el viernes pedagógico de Junio de 2010, se adelantó la realización de talleres sobre los pilares de Arte y Juego, y se convino que:

\footnotetext{
"Estos pilares fueran trabajados con talleres que ampliaran la mirada sobre estos y no se redujeran al trabajo de rincones y talleres como estrategias pedagógicas, que ya durante el año 2009 estas estrategias ya habían sido trabajadas en los jardines" (SDIS-UPN, 2012a, p. 17).
}

En Julio, se trabajó el pilar de Exploración del medio y Literatura, y para el segundo semestre de 2010 se desarrolló un trabajo que apuntara a la elaboración del encuentro de Maestras Enseñan a Maestras (MEM), que consiste en la elección de experiencias pedagógicas de los jardines infantiles para ser compartidas entre los mismos jardines.

En septiembre y octubre de 2011 se llevó a cabo la realización de talleres orientados por el Equipo de Asesoría, el Equipo de Inclusión y el Equipo Pedagógico, que mantuvieron las temáticas vinculadas con los pilares de la educación inicial, abordando: Literatura, Expresión Corporal, Exploración del medio y Expresión Plástica (SDIS-UPN, 2012a). En noviembre realizó el encuentro MEM en su versión 2011, que contó con algunas experiencias desde el arte y la literatura.

\section{Conferencias Masivas}

Las conferencias masivas son una propuesta dentro de la estrategia de Formación y actualización del Equipo de Asesoría, que funcionó como un mecanismo para lograr un mayor impacto en la formación sobre educación inicial. Se quiso que a partir de estos encuentros, las maestras tuvieran un interés mayor en profundizar en diferentes temáticas. Se trataba de un ciclo de conferencias masivas en el que se convocó "a maestras, maestros, coordinadores y coordinadoras de los jardines infantiles para brindar información $\mathrm{y}$ experiencias que permitan reflexionar sobre temas relacionados con primera infancia y que fortalezcan la cualificación de la educación inicial" (SDIS, 2012, p. 97).

Las conferencias propuestas han sido realizadas por expertos en los temas de arte, inclusión y diversidad. Metodológicamente algunas se realizaron a manera de conferencia y en otras ocasiones como conferencia taller.

\section{Experiencia de acompañamiento UPN-SDIS}

Este apartado tiene en cuenta la participación de los autores del artículo ${ }^{9}$ en los contratos interinstitucionales celebrados entre la SDIS y la UPN, así como el Informe Final del segundo contrato finalizado en abril del 2012 (SDIS-UPN, 2012b).

9 José Ignacio Galeano en los dos contratos interadministrativos entre la SDIS y la UPN, formó parte de los equipos constituidos para el acompañamiento directo a jardines infantiles. Santiago Barbosa fue supervisor del segundo convenio interadministrativo y además cumplió el rol de invitado como experto en el trabajo de experiencias en torno al arte en la educación inicial. Durante el período de este trabajo ambos profesionales estaban vinculados al Equipo Pedagógico Central de la SDIS. 


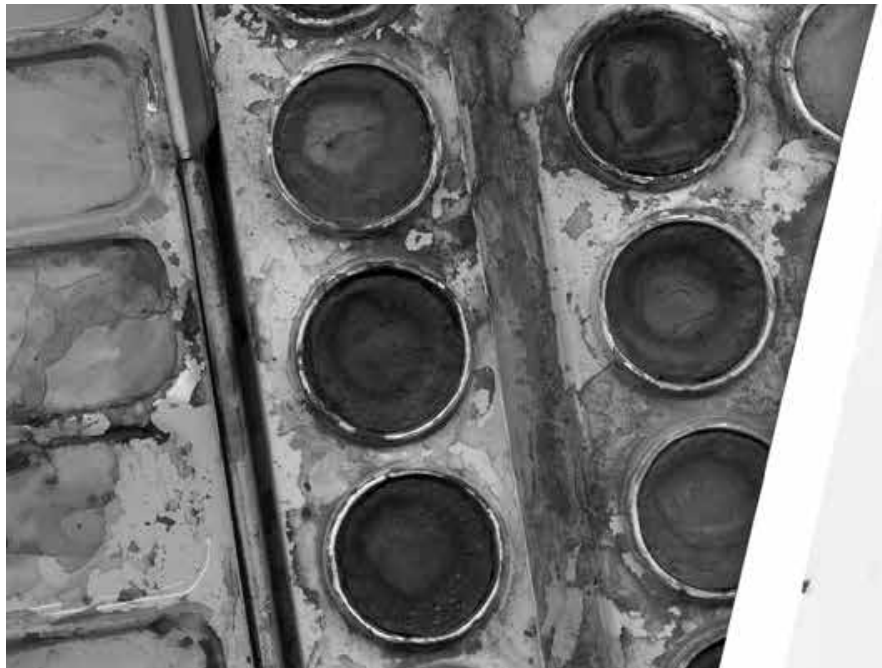

Dos convenios interinstitucionales SDIS-UPN tuvieron como uno de sus propósitos el trabajo de acompañamiento a jardines infantiles para cualificar sus prácticas pedagógicas. El proceso de acompañamiento tuvo dentro de sus objetivos apoyar la reformulación de los proyectos pedagógicos, en un primer convenio, y trabajar alrededor de los pilares de la educación inicial, en el segundo. El trabajo se estructuró a partir de la conformación de equipos integrados por profesionales de la SDIS y docentes de la UPN que irían a los jardines infantiles, trabajando alrededor de diferentes estrategias.

A continuación se desatacan un par de experiencias derivadas del segundo contrato. En algunos talleres referidos a la literatura infantil, se propuso a los jardines trabajar alrededor de las diferentes posibilidades que tiene la literatura. Se hicieron reflexiones sobre lectura de imágenes, la importancia de jugar con los sonidos del lenguaje, el hecho de que la literatura infantil va más allá de las palabras, el disfrute por el juego con los sonidos y las melodías, la lectura en voz alta y las posibilidades de trabajar con sombras chinescas, dramatizaciones o láminas. Las propuestas, en general, se desarrollaron desde los cuentos, las posibilidades sonoras del lenguaje, la lectura de imágenes, todo desde el diseño de ambientes (SDIS-UPN, 2012b).

Otra vivencia, tuvo que ver con un jardín infantil cuyo proyecto pedagógico se centraba en el arte. Una maestra licenciada en artes lideraba el trabajo referido al arte y dirigió un

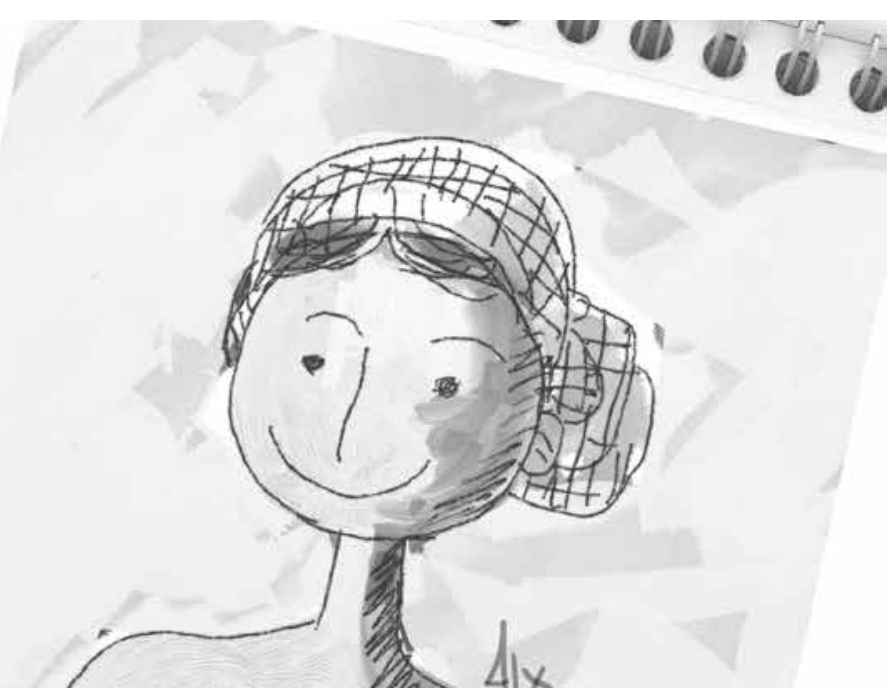

taller a manera de recorrido a través de la danza, el teatro y la plástica. Se realizó otro trabajo con el jardín respecto al acompañamiento en el desarrollo de su propuesta para el Encuentro de Maestras Enseñan a Maestras ${ }^{10}$, y del que se concluyó la existencia de una mirada del arte como escenario para la expresión, una apuesta por el arte como lenguaje, un reconocimiento de que el arte debe estar articulado a los ambientes y a los espacios ofrecidos a los niños. Se encuentra también que la expresión "técnica", como un aspecto del trabajo del arte en primera infancia, puede ser una limitante y contradicción con lo trabajado y el Lineamiento Curricular.

Al abordar el pilar de arte se contó con el apoyo de un experto. Se desarrollaron encuentros con los jardines infantiles que tuvieron como estructura (SDIS-UPN, 2012b):

- Un conversatorio acerca de cómo intervenir plásticamente un espacio, tomando como base imágenes y fotografías de intervenciones en el espacio realizadas por artistas contemporáneos.

- Una organización por grupos que eligieron un espacio del jardín y materiales para realizar una instalación, pensando en una intervención que genere interacciones con el espa-

10 El Encuentro de Maestras Enseñan a Maestras es un evento llevado a cabo en los años 2010 y 2011, en el que los jardines tienen la oportunidad de compartir su trabajo con otros jardines infantiles. 
cio, juego y exploración. En algunas de las zonas diseñadas se quiso brindar posibilidades a los niños y niñas de reconocer diferentes sonidos, acercarse a texturas, evidenciar diferentes fenómenos físicos al movimiento de los objetos, diferentes ajustes posturales, posibilidades de explorar y jugar con materiales y con el espacio. En otras áreas se pretendió posibilitar la exploración del espacio, diferentes sensaciones al paso entre las estructuras, posibilidades de continuar transformando el espacio y creando nuevas construcciones, diferentes movimientos y desplazamientos.

- Reflexión sobre cada espacio, reconociendo el proceso vivido para su instalación y las posibilidades pedagógicas de los dos ambientes.

En este trabajo con los jardines y sus maestras ocurrieron desplazamientos alrededor de algunas ideas que van a señalar el camino de sus intervenciones pedagógicas respecto al arte. Este es el caso del reiterado privilegio que se le daba a la técnica artística sobre el proceso. En un comienzo primó la idea de que en el trabajo con los niños algo fundamental sería terminar el producto a partir del dominio de una forma de expresión artística (modelado, la pintura).

Las interacciones dejaron ver que esto tiene una importancia relativa, pero que exista expresión, que el niño establezca interacciones con los materiales, se sensibilice ante las diferentes manifestaciones artísticas, o simplemente quiera dejar huella a partir de la utilización de materiales variados, también le otorga sentido al arte y lo trasciende a una aproximación exclusivamente disciplinar.

Los espacios y ambientes resultaron ser otro importante desplazamiento. El arte no está solamente en la mesa de trabajo de los niños, sino que está presente en el espacio y en los distintos ambientes del jardín. El trabajo en la intervención del espacio o en la creación de ambientes para los niños, bien puede constituirse en otra manera de hacer arte en los jardines. Así, la maestra hace arte para invitar a los niños a que interactúen con él. El diseño de un ambiente bien puede ser una "composición", con la firme intención de ofrecer un ambiente pedagógico que invite al trabajo y sugiera actividad (SDIS-UPN, 2012b).

\section{Equipo de Arte y Literatura}

Desde la aparición del Lineamiento Pedagógico y su posterior posicionamiento, comenzó a presentarse cada vez más una mayor intención por promover el trabajo pedagógico relacionado con el arte en la Educación Inicial. Los procesos de formación y acompañamiento dirigidos a las maestras de los jardines infantiles: espacios de viernes pedagógicos, experiencias de acompañamiento UPN-SDIS, proceso de posicionamiento del Lineamiento Pedagógico y conferencias masivas, propuestas por el Equipo de Asesoría, no lograban dar aún un cubrimiento acorde con la magnitud de las circunstancias. De dicha inquietud surgió la idea de conformar un equipo que se enfoque de manera específica en la formación y asesoría a maestras sobre la implementación del arte en la educación inicial.

De acuerdo con lo anterior, el equipo de Arte deviene de una propuesta presentada por un grupo inicial de cinco personas pertenecientes al Equipo Pedagógico con formación y experiencia en arte y educación inicial. La propuesta, aprovechar el perfil profesional de este grupo, además de su experiencia de trabajo, para poder llevar a cabo una labor de formación, asesoría, acompañamiento y movilización social desde el arte en la Educación Inicial y en la primera infancia.

Durante el último trimestre de 2012 se llevó a cabo el piloto de un ciclo de formación general en arte, el cual estuvo dirigido a maestras y maestros de jardines infantiles de la SDIS, para tal fin se abrió una convocatoria con inscripciones virtuales vía internet y con un total de aproximadamente ciento veinte cupos, divididos en cuatro subgrupos de treinta cupos por punto o zona de confluencia (localidades de Kennedy, San Cristóbal sur, Usaquén y Barrios Unidos), que ofrecía formación y asesoría en lo concerniente a experiencias artísticas en Educación Inicial, desde lo plástico, lo corporal, lo musical y lo literario, no por ello dejando de contemplar para un futuro próximo también lo dramático.

El objetivo fue que cada participante, sin importar la zona en la que se inscriba, pueda participar de una sesión cada semana durante un mes, de cada uno de los talleres diseñados para cada experiencia, y que de acuerdo a sus intereses personales así como a las expectativas del jardín del que forme parte, pueda escoger en cual de dichas experiencias quisiera profundizar; para dar continuidad a lo anterior se pensó en llevar a cabo un ciclo de profundización. 


\section{Los espacios de formación propuestos mantuvieron la idea de taller y, como tal, se quiso incorporar un fuerte componente desde el hacer"}

Cada taller del ciclo general en arte, tiene como premisa el ofrecer una experiencia artística cotidiana y de reflexión directa de su sentido e intencionalidad, sin que se tenga que "infantilizar" la experiencia, sino, por el contrario, teniendo como objetivo la reflexión desde la vivencia adulta, tanto, como para tener consciencia de que el desarrollo artístico es algo humano y no es exclusivo de los artistas, y poder entender que no existe una edad determinada para ser conscientes de ello. Esto posibilitará que las maestras puedan decantar su propia experiencia a favor de la generación de propuestas pensadas para niños y niñas de 0 a 5 años de edad.

Los espacios de formación propuestos mantuvieron la idea de taller y, como tal, se quiso incorporar un fuerte componente desde el hacer. Sin embargo, también se planteó la idea de incluir espacios permanentes de reflexión bajo el diálogo de saberes. De este modo, se partió de conversar alrededor de las experiencias de vida en relación con el arte (infancia, escuela, trabajo en educación infantil), del intercambio desde preguntas claves, de la vivencia de experiencias que vinculen el arte plástico, la música, la literatura o lo corporal, y de la reflexión a partir de lo vivido.

\section{Proyecciones y conclusiones}

El resultado de este piloto demostró el gran y creciente interés de maestros y maestras por aproximarse, experimentar y entender cómo abordar el arte en la primera infancia y en la educación inicial. Por ello, y como parte de la propuesta del Equipo de Arte, se pretende generar espacios de profundiza- ción a modo de ciclos de formación en las diferentes experiencias artísticas. Esto con el propósito de articular la formación a los intereses de las maestras y maestros. Lo anterior tiene como valor agregado la posibilidad de generar un proceso de acompañamiento en la práctica que permita una cercanía con la realización de las propuestas pedagógicas de las maestras y el trabajo de asesoramiento desde el Equipo de Arte.

La propuesta del equipo incluye también una articulación con instancias intra-institucionales e interinstitucionales, con el fin de poder generar una movilización social desde el arte. Esta apuesta de trabajo articulado mantiene coherencia con la apuesta distrital para la primera infancia. Pero, además de la voluntad política, este trabajo reconoce la necesidad de concebir el arte como una vivencia al alcance de diferentes grupos poblacionales que tienen que ver con la infancia y que puede transformar la mentalidad y el actuar de las personas con respecto a los niños y las niñas, porque el arte se constituye también en un lenguaje que posibilita nuevas sensibilidades y comprensiones de la realidad.

Por último, se considera como necesario ampliar la formación para incluir las experiencias artísticas de danza y de arte dramático, escenarios en los que no se ha puesto énfasis desde los procesos adelantados en los últimos años. Por ello, y teniendo en cuenta la inmensa cantidad de maestras susceptibles de participar en estos procesos de formación, ha de requerirse el fortalecimiento del equipo de arte, aumentando el número de profesionales, pero además ampliando el espectro de sus formaciones y experiencias profesionales. 


\section{Referencias}

Bonnafé, M. (2008). Leer, eso es bueno para los bebés. México: Océano.

Departamento Administrativo de Bienestar Social, DABS. (2000). Proyecto Pedagógico Red de Jardines Sociales. Bogotá: Quebecor impreandes.

Departamento Administrativo de Bienestar Social, DABS. (2003). Desarrollo infantil y Educación Inicial: Avances del proyecto pedagógico del DABS. Bogotá: Quebecor World Bogotá.

Fandiño, G. (2012, S.P.). Algunas reflexiones sobre el arte en el arte en la primera infancia y el papel del sector cultura en ellos. Bogotá.

Hoyuelos, A. (2006). La estética en el pensamiento y obra pedagógica de Loris Malaguzzi. Barcelona: Octaedro, Rosa Sensat.

Ministerio de Educación Nacional, MEN. (2012, S.P.). Una propuesta pedagógica para la primera infancia. Documento Base para la construcción del Lineamiento Pedagógico de Educación Inicial Nacional. República de Colombia.

Reyes, Y. (2007). La casa imaginaria. Lectura y literatura en la primera infancia. Bogotá: Norma.

Secretaría Distrital de Integración Social, SDIS - Equipo Pedagógico y Equipo de Asesoría. (2009). Documento de circulación interna SDIS. Orientaciones para la estructuración y/o revisión del proyecto pedagógico. Bogotá: SDIS.

Secretaría Distrital de Integración Social, SDIS - Equipo de Asesoría a Servicios Sociales de Infancia en el Estándar Proceso Pedagógico. (2012). Documento de circulación interna SDIS. Aproximación a la sistematización de la experiencia de la Secretaría Distrital de Integración Social en procesos de asesoría y acompañamiento pedagógico. Bogotá: SDIS.

Secretaría Distrital de Integración Social, SDIS y Secretaría de Educación del Distrito, SED. (2010). Lineamiento pedagógico y curricular para la educación inicial en el Distrito Capital. Bogotá: Imprenta Nacional de Colombia.

Secretaría Distrital de Integración Social, SDIS y Universidad Pedagógica Nacional, UPN. (2012a). Balance Viernes Pedagógico 2008 - 2012. Bogotá.

Secretaría Distrital de Integración Social, SDIS y Universidad Pedagógica Nacional, UPN. (2012b). Informe final contrato interadministrativo 3643. Bogotá.

Vigottsky, L. (1996). La imaginación y el arte en la infancia. México: Akal. 